Article

\title{
Crystallization and Crystallographic Analysis of a Bradyrhizobium Elkanii USDA94 Haloalkane Dehalogenase Variant with an Eliminated Halide-Binding Site
}

\author{
Tatyana Prudnikova ${ }^{1,2,+}$, Barbora Kascakova ${ }^{1,+}$, Jeroen R. Mesters ${ }^{3}$ D , Pavel Grinkevich ${ }^{1}$, \\ Petra Havlickova ${ }^{1}$, Andrii Mazur 1,2, Anastasiia Shaposhnikova 1,2 ${ }^{1 D}$, Radka Chaloupkova ${ }^{4}$, \\ Jiri Damborsky ${ }^{4,5}$, Michal Kuty ${ }^{1,2}$ and Ivana Kuta Smatanova ${ }^{1,2, * \mathbb{D}}$ \\ 1 Faculty of Science, University of South Bohemia in Ceske Budejovice, Branisovska 1760, 37005 Ceske \\ Budejovice, Czech Republic \\ 2 Center of Nanobiology and Structural Biology, Institute of Microbiology of the Czech Academy of Sciences, \\ Zamek 136, 37333 Nove Hrady, Czech Republic \\ 3 Institute of Biochemistry, University of Lübeck, Ratzeburger Allee 160, 23538 Lübeck, Germany \\ 4 Loschmidt Laboratories, Department of Experimental Biology and RECETOX, Faculty of Science, Masaryk \\ University, Kamenice 5/A4, 62500 Brno, Czech Republic \\ 5 International Clinical Research Center, St. Anne's University Hospital Brno, Pekarska 53, \\ 65691 Brno, Czech Republic \\ * Correspondence: ivanaks@seznam.cz or talianensis@gmail.com \\ + These authors contributed equally to this work.
}

Received: 27 June 2019; Accepted: 19 July 2019; Published: 23 July 2019

\begin{abstract}
Haloalkane dehalogenases are a very important class of microbial enzymes for environmental detoxification of halogenated pollutants, for biocatalysis, biosensing and molecular tagging. The double mutant (Ile44Leu + Gln102His) of the haloalkane dehalogenase DbeA from Bradyrhizobium elkanii USDA94 (DbeA $\Delta \mathrm{Cl}$ ) was constructed to study the role of the second halide-binding site previously discovered in the wild-type structure. The variant is less active, less stable in the presence of chloride ions and exhibits significantly altered substrate specificity when compared with the DbeAwt. DbeA $\Delta \mathrm{Cl}$ was crystallized using the sitting-drop vapour-diffusion procedure with further optimization by the random microseeding technique. The crystal structure of the DbeA $\Delta \mathrm{Cl}$ has been determined and refined to the $1.4 \AA$ resolution. The DbeA $\Delta \mathrm{Cl}$ crystals belong to monoclinic space group $\mathrm{C} 121$. The DbeA $\Delta \mathrm{Cl}$ molecular structure was characterized and compared with five known haloalkane dehalogenases selected from the Protein Data Bank.
\end{abstract}

Keywords: Haloalkane dehalogenase; halide-binding site; random microseeding

\section{Introduction}

Hazardous halogenated compounds are an important class of environmental pollutants. An obvious critical step in the potential biodegradation pathway is the dehalogenation process [1,2]. Haloalkane dehalogenases (HLDs) play an essential role in biodegradation of the halogenated pollutants. HLDs are predominantly bacterial enzymes that belong to the superfamily of $\alpha / \beta$-hydrolases and catalyze the hydrolytic conversion of a wide range of halogenated aliphatic compounds, and therefore play an important role in bioremediation [3] and industrial biocatalytic processes [4]. An aliphatic alcohol, a halide and a hydrogen cation are released during the enzymatic dehalogenation of haloalkanes by HLDs. The tertiary structures of HLDs are composed of a conserved $\alpha / \beta$-hydrolase core domain and an $\alpha$-helical cap domain [5]. The core domain is responsible for the catalytic reaction of the enzyme 
and the cap domain is essential for substrate specificity and recognition [6]. A deep cleft is situated between these two domains, allowing the solvent to access the buried active site. The active site is composed of two halide-anion stabilizing residues and the catalytic triad consisting of a nucleophile, a base and an acid [7]. HLDs can be divided into three subfamilies, HLD-I, HLD-II and HLD-III, according to the composition of the catalytic residues and the anatomy of the cap domain [4].

A novel HLD DbeA from B. elkanii USDA94, a member of HLD-II subfamily [4], was structurally and biochemically characterized [7]. The structure of DbeA wild type was determined to $2.2 \AA$ resolution and displays a typical topology of the $\alpha / \beta$-hydrolases (EC 3.8.1.5). The unique feature of the DbeA structure is the presence of two halide-binding sites, both fully occupied by chloride anions [7]. The first halide-binding site is located in the protein active site and is involved in substrate binding and stabilization of halogen ion produced during dehalogenation reaction. DbeA active site consists of five catalytic residues: two halide stabilizing residues (Trp104 and Asn38) and three amino acids essential for the catalytic activity of the enzyme [2,4]: the nucleophile Asp103, the catalytic base His271, and the catalytic acid Glu127. The second halide-binding site in DbeA is unique and has never been observed within HLD structures deposited in the PDB [8]. The second halide-binding site, which is buried in the protein core domain and located approximately $10 \AA$ far from the first halide-binding site, is formed by five amino-acid residues: Ile44, Gln274, Gln102, Gly37 and Thr40 [7]. Superposition of the DbeA structure with other related HLD-II members revealed the presence of two unique amino acids in the second halide-binding site: Gln102 instead of a typical His and Ile44 as a substitution of an ordinary Leu, thereby sufficiently increasing the cavity volume to accommodate the second halide ion. The variant DbeA $\Delta \mathrm{Cl}$ (Ile44Leu+Gln102His) was constructed and biochemically characterized to elucidate the role of the second halide-binding site in structure and function of DbeA [7].

Removal of the second halide binding site in DbeA significantly changed the substrate specificity of DbeA $\Delta \mathrm{Cl}$ and reduced the catalytic activity by an order of magnitude towards most of the tested substrates [7]. Wild-type DbeA is more active, its melting temperature rises with an increasing concentration of chloride salts and the binding energy for chloride ions is higher when compared with the DbeA $\Delta \mathrm{Cl}$ variant [7]. It was suggested that the chloride anion bound in a vicinity of second binding site may increase basicity of catalytic histidine and consequently accelerate the nucleophilic addition of water to the alkyl-enzyme intermediate [7]. In previous attempts, the crystallization of DbeA $\Delta \mathrm{Cl}$ was unsuccessful. The obtained crystals were very unstable, sensitive to mechanical stress and poorly diffracted X-rays to about $10 \AA$ A resolution. It took several years to grow crystals with an improved diffraction quality. Here, we report the successful crystallization, structure determination and further characterization of DbeA $\Delta \mathrm{Cl}$ variant.

\section{Materials and Methods}

\subsection{Gene Synthesis, Cloning, Expression and Protein Purification}

The recombinant gene dbeA $\Delta$ Cl-His6 (Ile44Leu + Gln102His) was synthesized artificially (Entelechon, Regensburg, Germany) according to the DbeA sequence [7] (Table 1). The restriction endonucleases NdeI and XhoI (Fermentas, Burlington, Canada) and T4 DNA ligase (Promega, Madison, USA) were applied to transfer the synthesized gene into the expression vector pET-21b (Novagen, Madison, USA). In order to overexpress DbeA $\Delta \mathrm{Cl}$ in E. coli BL21(DE3) cells, the final genes were transcribed by T7 RNA polymerase, which is expressed by the isopropyl $\beta$-D-1-thiogalactopyranoside (IPTG)-inducible lac UV5 promoter. Cells containing the plasmid were cultured in Luria broth medium at $310 \mathrm{~K}$. When the culture reached an optical density of 0.6 at a wavelength of $600 \mathrm{~nm}$, gene expression (at $293 \mathrm{~K}$ ) was induced by the addition of $0.5 \mathrm{mM}$ IPTG. The cells were subsequently harvested and disrupted by sonication using a Soniprep 150 (Sanyo Gallenkamp PLC, Loughborough, England). The supernatant was collected after centrifugation at $100,000 \mathrm{~g}$ for $1 \mathrm{~h}$. The crude extract was further purified on a HiTrap Chelating HP $5 \mathrm{ml}$ column charged with $\mathrm{Ni}^{2+}$ ions (GE Healthcare, Uppsala, Sweden). The His-tagged enzyme was bound to the resin in the presence of $20 \mathrm{mM}$ potassium phosphate buffer 
$\mathrm{pH} 7.5,0.5 \mathrm{M}$ sodium chloride, $10 \mathrm{mM}$ imidazole. Unbound and non-specifically bound proteins were washed out by a buffer containing $37.5 \mathrm{mM}$ imidazole. The target enzyme was eluted with a buffer containing $300 \mathrm{mM}$ imidazole. The active fractions were pooled and dialyzed overnight against $50 \mathrm{mM}$ Tris- $\mathrm{HCl} \mathrm{pH}$ 7.5. The DbeA $\Delta \mathrm{Cl}$ enzyme was stored at $277 \mathrm{~K}$ in $50 \mathrm{mM}$ Tris- $\mathrm{HCl} \mathrm{pH} 7.5$ buffer prior to analysis. The DbeA $\Delta \mathrm{Cl}$ production information is summarized in Table 1.

Table 1. Production specifics for DbeA $\Delta \mathrm{Cl}$.

\begin{tabular}{cc}
\hline Source Organism & Bradyrhizobium Elkanii USDA94 \\
\hline DNA source & Artificially synthesized DNA \\
\hline Transport vector & pMA \\
\hline Expression vector & pET-21b \\
\hline Expression host & E. coli BL21(DE3) \\
\hline Complete amino acid sequence of & FMEFIRPMRDWSDFHQHDAARETFRKFRTPGVGEAMILDN \\
DbeA $\triangle \mathrm{Cl}$ & NAFVERVLPGSILRTLSEEEMAAYRAPFATRESRMPTLML \\
& PRELPIAGEPADVTQALTAAHAALAASTYPKLLFVGSPGA \\
& LVSPAFAAEFAKTLKHCAVIQLGAGGHYLQEDHPEAIGRS \\
& VAGWIAGIEAASAQRHAALEHHHHHH \\
\hline
\end{tabular}

\subsection{Crystallization}

The freshly isolated and purified DbeA $\Delta \mathrm{Cl}$ protein was crystallized at a concentration of $30 \mathrm{mg} \cdot \mathrm{ml}^{-1}$ in $50 \mathrm{mM}$ Tris- $\mathrm{HCl}$ buffer $\mathrm{pH} 7.5$ by the sitting-drop vapour-diffusion procedure [9]. For initial screening several commercial precipitants kits were used: JBScreen Classic Kits № 1-10 and Wizard I-III (Jena Bioscience GmbH, Jena, Germany), Morpheus®HT-96, JCSG-plus ${ }^{\mathrm{TM}}$ HT-96, PACT premier ${ }^{\mathrm{TM}}$ HT-96 and Structure Screen $1+2$ HT-96 kit (Molecular Dimensions Ltd (MDL), Suffolk, UK), Crystal Screen kit, PEGRx HT ${ }^{\mathrm{TM}}$ and PEG/Ion HT ${ }^{\mathrm{TM}}$ (Hampton Research (HR), Aliso Viejo, USA) and Axygen I-VIII crystallization kits (Axygen Biosciences, Union City, USA). The CombiClover 96 well plates (MDL, Suffolk, UK) for manual screening experiments as well as Swissi polystyrene MRC 2-drop plate (MDL, Suffolk, UK) were utilized for the initial screening on an Oryx3 robot (Douglas Instruments Ltd, Hungerford, UK) for the DbeA $\Delta \mathrm{Cl}$ protein.

The hanging drop crystallization trials were carried out in Limbro 24 well plates (HR, Aliso Viejo, USA). The Douglas Instruments, USA Vapour Batch 96 well plates were used to perform the microbatch under oil crystallization [10]. Macroseeding experiments [11] were carried out by lowering the protein concentration to $20-25 \mathrm{mg} \cdot \mathrm{ml}^{-1}$. The counter-diffusion crystallization was performed in single glass capillaries with inner diameters ranging from 0.1 to $0.4 \mathrm{~mm}$ (HR, Aliso Viejo, USA) using a three-layer configuration [12].

\subsection{Data Collection, Processing and Structure Solution}

X-ray diffraction data at $100 \mathrm{~K}$ were collected to the $1.4 \AA$ resolution at the BESSY II electron storage ring on beamline MX 14.1 of the Helmholtz-Zentrum Berlin (Berlin-Adlershof, Germany; [13]). 2500 images were processed with the graphical user interface XDSAPP [14] for running XDS [15]. Phasing by molecular replacement was performed using MOLREP [16] and the structure of DbeA as the template (PDB code 4k2a; [7]). One molecule was found in the asymmetric unit of DbeA $\Delta \mathrm{Cl}$. Structure refinement and model building was performed using isotropic and anisotropic refinement protocols in REFMAC5 [17] and Coot [18] from the CCP4 package [19], respectively. The quality of the protein models was confirmed with MolProbity [20,21] and wwPDB [22] validation servers. The structure of the DbeA $\Delta \mathrm{Cl}$ has been deposited to the Protein Data Bank under accession code $6 s 42$. 
Figures with the structure were prepared using the program PyMOL [23]. The complete information about the data collection, processing, and refinement statistics are provided in Table 2.

Table 2. Data collection and crystallographic statistics.

\begin{tabular}{|c|c|}
\hline \multicolumn{2}{|c|}{ X-ray Diffraction Data Collection Statistics } \\
\hline Space group & $\mathrm{C} 121$ \\
\hline Cell parameters $(\AA, \circ)$ & $a=128.95, b=63.95, c=46.05 ; \alpha=\gamma=90, \beta=106.27$ \\
\hline Wavelength $(\AA)$ & 0.918 \\
\hline Resolution $(\AA)$ & 1.39 \\
\hline Number of unique reflections & 68,322 \\
\hline Redundancy & $2.18(2.20)$ \\
\hline Completeness (\%) & $96.13(92.17)$ \\
\hline$R_{\text {merge }} \#$ & $4.9(26.3)$ \\
\hline Average $\mathrm{I} / \sigma(\mathrm{I})$ & $12.72(3.19)$ \\
\hline Wilson B $\left(\AA^{2}\right)$ & 20.8 \\
\hline \multicolumn{2}{|c|}{ Refinement Statistics } \\
\hline Resolution range $(\AA)$ & $41.96-1.4(1.43-1.39)$ \\
\hline No. of reflections in working set & $64,907(4,609)$ \\
\hline R value (\%) \#\# & 13.98 \\
\hline $\mathrm{R}_{\text {free }}$ value $(\%)$ & 15.22 \\
\hline RMSD bond length $(\AA)$ & 0.006 \\
\hline RMSD angle $\left(^{\circ}\right)$ & 1.635 \\
\hline No. of atoms in AU & 2,823 \\
\hline No. of protein atoms in AU & 2,333 \\
\hline No. of water molecules in AU & 470 \\
\hline No. of iodide ions in AU & 8 \\
\hline No. of chloride ions in AU & 3 \\
\hline Mean B value $\left(\AA^{2}\right)$ & 13.42 \\
\hline \multicolumn{2}{|c|}{ Ramachandran Plot Statistics } \\
\hline - Residues in favoured regions (\%) & 97.2 \\
\hline - Residues in allowed regions (\%) & 100 \\
\hline PDB code & $6 s 42$ \\
\hline
\end{tabular}

The data in parentheses refer to the highest-resolution shell. ${ }^{\#} R_{\text {merge }}=\left(\left|I_{h k l}-\langle I\rangle\right|\right) / I_{h k l}$, where the average intensity $\langle\mathrm{I}\rangle$ is taken over all symmetry equivalent measurements and $\mathrm{I}_{\mathrm{hkl}}$ is the measured intensity for any given reflection; \#\#-value $=\|\left|\mathrm{F}_{\mathrm{o}}\right|-\left|\mathrm{F}_{\mathrm{c}}\right| /\left|\mathrm{F}_{\mathrm{o}}\right|$, where $\mathrm{F}_{\mathrm{o}}$ and $\mathrm{F}_{\mathrm{c}}$ are the observed and calculated structure factors, respectively; $\# R_{\text {free }}$ is equivalent to $R$ value but is calculated for $5 \%$ of the reflections chosen at random and omitted from the refinement process.

\section{Results and Discussion}

The crystallization procedure previously successfully used for growing of the DbeAwt protein [24] was applied to prepare crystals of freshly purified DbeA $\Delta \mathrm{Cl}$ protein. Initially, only a light amorphous precipitation was observed. Further optimization of the crystallization conditions was carried out by variation of the protein and precipitant concentrations (PEG and salt) and drop protein-reservoir ratio composition. This optimization did not improve the results. Additional screening was conducted by applying further commercial crystallization kits: JBScreen Classic Kits № 1-10 and Wizard I-III (Jena Bioscience $\mathrm{GmbH}$, Jena, Germany). Again, only very small and thin needle crystals or a heavy amorphous precipitation were observed. The next optimization step was based on moving the system closer to the metastable zone based on the phase transition diagram by decreasing the concentration of the crystallization drop components. The reduction of the DbeA $\Delta \mathrm{Cl}$ concentration and variation of the precipitant concentrations yielded small microcrystals (Figure 1a) and a two-dimensional (2D) single needle crystals (Figure 1b) within a period of 1-3 weeks. The microcrystals were grown within three weeks from precipitant consisting of $28 \%$ (w/v) PEG 4000, $0.2 \mathrm{M} \mathrm{Li}_{2} \mathrm{SO}_{4}$ in $0.1 \mathrm{M}$ Tris $\mathrm{pH} 8.2$ buffer and a $30 \mathrm{mg} \cdot \mathrm{ml}^{-1}$ protein concentration. The small needle crystals were observed after 10 days at a 10 to 
$30 \mathrm{mg} \cdot \mathrm{ml}^{-1}$ protein concentration in precipitant composed of $12-17 \%(\mathrm{w} / \mathrm{v})$ PEG 3350, 115- $125 \mathrm{mM}$ $\mathrm{MgCl}_{2}$ in $100 \mathrm{mM}$ Tris-HCl 7.5 buffer.

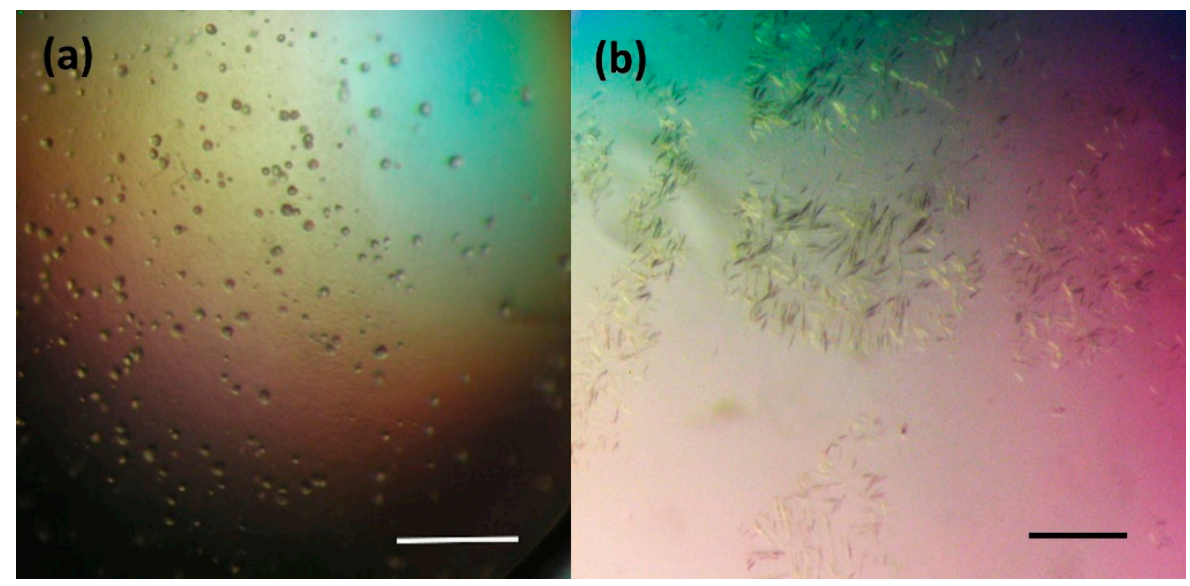

Figure 1. Results of initial crystallization experiments of $\mathrm{DbeA} \Delta \mathrm{Cl}$ protein from B. elkanii USDA94:

(a) microcrystals and (b) small 2D needle crystals. The scale bar represents $100 \mu \mathrm{m}$.

In order to improve the crystal quality, Morpheus®HT-96, JCSG-plus ${ }^{\mathrm{TM}} \mathrm{HT}-96$, PACT premier ${ }^{\mathrm{TM}}$ HT-96 and Structure Screen $1+2$ HT-96 kit (Molecular Dimensions Ltd (MDL), Suffolk, UK), Crystal Screen kit, PEGRx HTTM and PEG / Ion HT ${ }^{\mathrm{TM}}$ (Hampton Research (HR), Aliso Viejo, USA) and Axygen I-VIII crystallization kits (Axygen Biosciences, Union City, USA) were applied. Finally, small 3D crystals (Figure 2a) with a dimension of about $15 \times 5 \times 35 \mu \mathrm{m}$ were grown from a solution containing $26.57 \%(\mathrm{w} / \mathrm{v})$ hexanediol at $295 \mathrm{~K}$ over 10 days. The crystals diffracted X-rays to a maximum resolution of 8-10 $\AA$. The quality of these crystals did not allow to record good diffraction data and additional strategies were needed to improve the size and shape of obtained crystals.

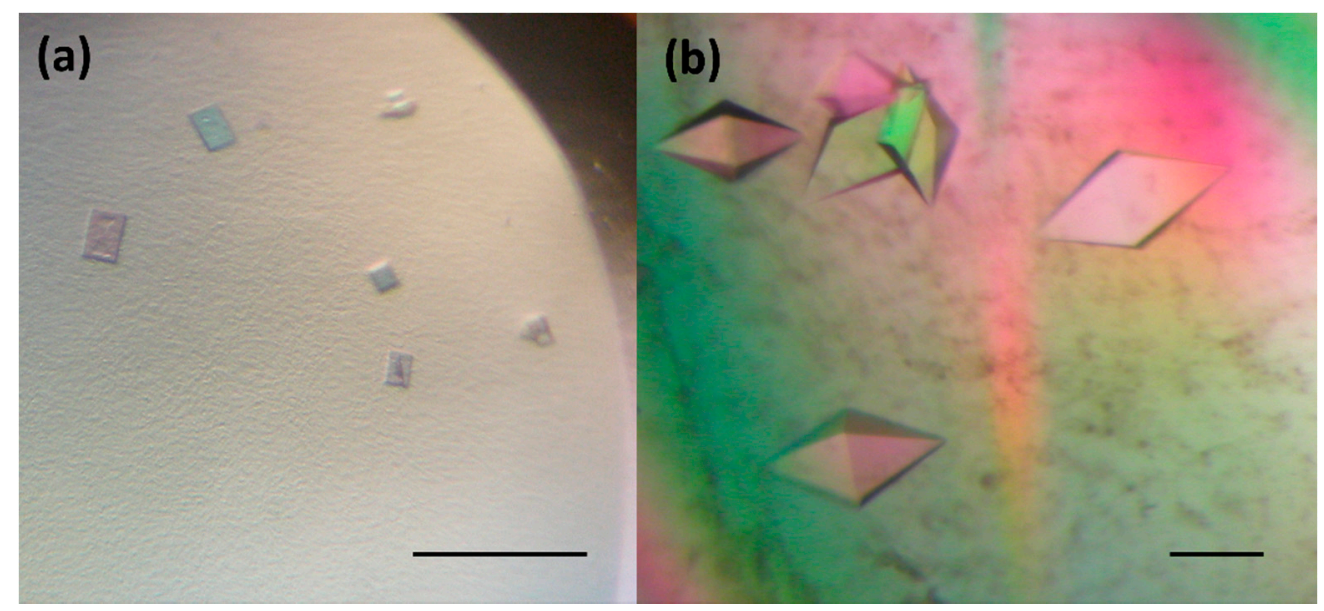

Figure 2. DbeA $\Delta \mathrm{Cl}$ crystals used for diffraction analysis: (a) small $3 \mathrm{D}$ crystals grown at $26.5 \%(\mathrm{w} / \mathrm{v}$ ) hexanediol and (b) big 3D crystals developed by random seeding experiments. The scale bar represents $100 \mu \mathrm{m}$. 
Further optimization using the precipitant solution mentioned above was pursued by setting up the experiments at a lower temperature (193K), by application of the Additive Screen (HR) and by variation of the protein concentration from 10 to $40 \mathrm{mg} \cdot \mathrm{ml}^{-1}$, however, without success. The same conditions were tested in a hanging drop vapour-diffusion, microbatch under oil, counter-diffusion and macroseeding procedure. All these experiments did not significantly improve the diffraction quality of the crystals. Next, the small crystals (Figure 2a) were used for random microseeding experiments [25] with application of two commercial crystallization kits: PACT premier ${ }^{\mathrm{TM}}$ HT-96 and Structure Screen $1+$ 2 HT-96 kit (MDL), which finally resulted in the appearance of 3D crystals with an average dimensions $70 \times 75 \times 100 \mu \mathrm{m}$ within a period of two weeks (Figure $2 \mathrm{~b}$ ). These crystals, later used for X-ray data collection experiments, were obtained by lowering the protein concentration to $20 \mathrm{mg} \cdot \mathrm{ml}^{-1}$ and applying the following precipitant: $0.2 \mathrm{M}$ sodium iodide and 20\% (w/v) PEG 3350.

Crystals of DbeA $\Delta \mathrm{Cl}$ diffracted X-rays to $1.4 \AA$ resolution and belong to the monoclinic base-centered space group $C 121$. The diffraction data allowed localizing 294 amino-acid residues fitting to the one molecule in the asymmetric unit. The overall shape of DbeA $\Delta \mathrm{Cl}$ is like a block with 2,823 non-hydrogen atoms ( 470 water molecules, 8 iodide anions, 3 chloride anions and hexanediol molecule) and corresponds to the canonical architecture of HLDs of the $\alpha / \beta$ hydrolase fold superfamily (Figure 3a). The structural organization of DbeA $\Delta \mathrm{Cl}$ displays two compact domains: an $\alpha / \beta$ hydrolase core domain and a helical cap domain with the active site located between them. The cap domain (residues 134-214) consists of five $\alpha$-helices $\left(\alpha 4, \alpha 5, \alpha 5^{\prime}, \alpha 6, \alpha 7\right.$ and $\left.\alpha 8\right)$ and six loops, together forming a lid, protecting the active site cavity. The core domain (residues 4-133 and 215-298) consists of a central twisted eight-stranded $\beta$-sheet with the $\beta 2$ strand running antiparallel. The $\beta$-sheet region is flanked by six $\alpha$-helices: two elements $(\alpha 1$ and $\alpha 2)$ cover one side and the remaining four $(\alpha 3, \alpha 9$, $\alpha 10$ and $\alpha 11$ ) the other side [2,26] (Figure 3a). The protein displays a monomer as the biological unit according to the analysis of crystal contacts between molecules in the unit cell and crystal packing. The exploration of macromolecular interfaces by PDBePISA server [27] underpins the monomeric nature of the protein.

The DbeA $\Delta \mathrm{Cl}$ active site displays a substrate-binding pocket typical for all haloalkane dehalogenases. The enzyme's active site cavity contains the catalytic triad consisting of Asp103, His 271 and Glu127. The nucleophile Asp103 is located at the turn between $\beta$-strand $\beta 5$ and helix $\alpha 3$. The catalytic base His 271 is positioned on the loop joining $\beta 8$ and $\alpha 11$. The catalytic acid Glu127 is located behind $\beta$-strand $\beta 6$. Inspecting the electron density map, one iodide anion and one molecule of hexanediol as components of precipitant cocktail were identified near the DbeA $\Delta \mathrm{Cl}$ active site. The iodide ion is mainly stabilized by interactions with the $\mathrm{N}$ atoms of two halide-binding residues: Asn34 $\mathrm{N}^{\delta 2}$ and Trp104 $\mathrm{N}^{\epsilon 1}$ with distances of $3.72 \AA$ and $3.47 \AA$, respectively. Further coordination is realized with the N atom of the pyrrolidine ring of Pro205 at a distance of $3.64 \AA$ and an $\mathrm{O}$ atom of bound hexanediol at $3.68 \AA$ A distance (Figure $3 b$ ).

The substitutions Ile44Leu and Gln102His introduced into the structure of DbeA result in the elimination of the second halide-binding site (Figure 3c). The site is occupied by a water molecule in the mutant enzyme. Apparently, insufficient space is left for the positioning of the second halide anion as observed in the DbeAwt structure. The atoms of three residues: Thr $40 \mathrm{O}^{\gamma 1}$, His $102 \mathrm{~N}^{\delta 1}$ and Gly37 N, with hydrogen bond distances of $2.74 \AA$, $2.79 \AA$, and $2.94 \AA$, respectively, coordinated water molecule W71 that was modelled instead of the halide anion (Figure 3c). Further coordination is realized by water molecule W11 at a distance of $2.73 \AA$. Water molecule W11 is situated between two halide-binding sites, $6.73 \AA$ away from the iodide ion in the canonical active site of the protein. Water W11 is stabilized by interaction with the $\mathrm{O}^{\delta 2}$ atom of catalytic nucleophile Asp103 at $2.71 \AA$ distance. 
(a)
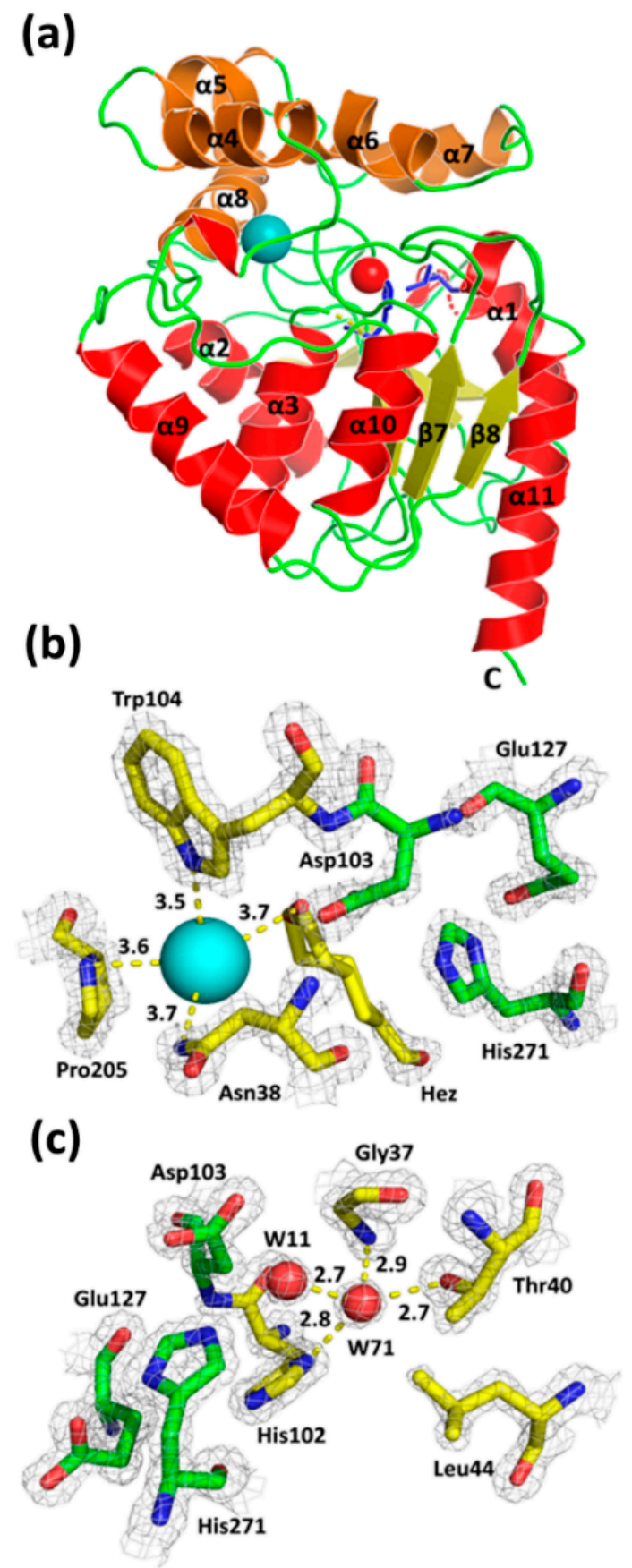

Figure 3. The overall structure of $\operatorname{DbeA} \Delta \mathrm{Cl}$ (a), close-up view of the canonical DbeA $\Delta \mathrm{Cl}$ active site (b) and second halide-binding site (c). The $2 \mathrm{~F}_{\mathrm{O}}-\mathrm{F}_{\mathrm{c}}$ electron-density map contoured at $2 \sigma$ is shown in grey (a) $C \alpha$ ribbon trace shows elements of the protein secondary structure. The $\alpha$-helices are coloured red for the main domain and brown for the cap domain; $\beta$-strands are coloured yellow; loops are shown in green; iodide ion is presented as a cyan sphere, water molecule (W71) is shown as a red sphere; the two point substitutions Ile44Leu + Gln102His introduced into DbeA are highlighted as blue sticks. (b) The iodide ion in the active site is presented as a cyan sphere with coordination interaction distances in $\AA$ and highlighted by yellow dashed lines; hexanediol (Hez) (shown in two alternative conformations) and amino acids Asp103, Trp104, Pro205 coordinating the iodide ion are shown as sticks with carbon atoms coloured yellow. Carbon atoms of catalytic triad are highlighted in green. (c) The water molecules W11 and W71 are presented as red spheres; amino acids coordinating water molecule W71 are shown as sticks with carbon atoms coloured in yellow with and interactions with distances in $\AA$ shown by yellow dashed lines. Water molecule W71 is located in second-halide binding site. 
The sequence of DbeA $\Delta \mathrm{Cl}$ from B. elkanii (PDB ID code: 6s42) was aligned (Figure 4) and compared with five known sequences of HLDs deposited in the PDB: DbeA wild type from B. elkanii (PDB ID code: 4k2a [7]), DhaA from Rhodococcus species (PDB ID code: 1bn6 [1]), DhlA from Xanthobacter autotrophicus (PDB ID code: 1cij [28]), LinB from Sphingomonas paucimobilis (PDB ID code: 1cv2 [29]) and DmbA from Mycobacterium tuberculosis (PDB ID code: 2qvb [30]). The reason for selection of these dehalogenases is that DbeAwt is the same protein without two point mutations occurring in DbeA $\Delta \mathrm{Cl}$ with $99.3 \%$ sequence identity, DhaA as HLD with highest sequence identity shows $50.7 \%$ and DhlA as HLD with lowest sequence identity displays $26.5 \%$ in comparison to DbeA $\Delta \mathrm{Cl}$. Alignment of DbeA $\Delta \mathrm{Cl}$ with different kind of haloalkane dehalogenase LinB from the same substrate specificity group (SSG-I) demonstrates $47.4 \%$ sequence identity similar to DmbA as another type of HLD from different SSG-III $(44.8 \%)$ [31].

\begin{tabular}{|c|c|c|}
\hline$\triangle \mathrm{Cl}$ & --VITISADISLHHRAVLGSTMAYRETGRSDAPH-VLELHGNPTSS 42 & 42 \\
\hline DbeAwt & ------- ADISLHHRAVLGSTMAYRETGRSDAPH-VLFLHGNPTSS 38 & 38 \\
\hline DhaA & 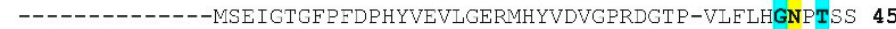 & 15 \\
\hline LinB & 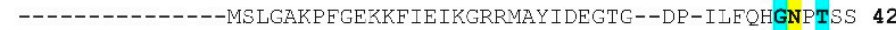 & 2 \\
\hline Dmba & $-------1-$ AFGVEPYGQPKYLEIAGKRMAYIDEGKG--DA-IVEQHGNPTSS 41 & \\
\hline \multirow[t]{2}{*}{ DhlA } & MVNAIRTPDQRFSNLDQYPESPNYLDDLPGYPGLRAHYLDEGNSDAEDVELCLHGEPTWS 60 & 0 \\
\hline & . $\quad * \quad * \quad * \quad * \quad .: * * * * *$ & \\
\hline DbeA $\triangle \mathrm{Cl}$ & YLWRNIMPLVAPVG-HCIAPDLIGYGQSGKPDI----SYRFFDQADYLDALIDELGIAS- 96 & 16 \\
\hline DbeAwt & YIWRNIMPLVAPVG-HCIAPDLIGYGQSGKPDI----SYRFFDQADYLDALIDELGIAS- 92 & \\
\hline DhaA & YLWRNI I PHVAPSH-RCIAPDLIGMGKSDKPDL----DYFFDDHVRYLDAF IEALGLEE- 99 & 9 \\
\hline LinB & YLWRN I MPHCAGLG-RLIACDLIGMGDSDKLDPSGPERYAYAEHRDYLDALWEALDLGDR 10 & 01 \\
\hline DmbA & YLWRNIMPHLEGLG-RLVACDLIGMGASDKLSPSGPDRYSYGEQRDFLEALWDALDLGDH 10 & .00 \\
\hline \multirow[t]{2}{*}{ DhlA } & YLYRKMIPVEAESGARVIAPDFFGFGKSDKPVD--EEDYTFEFHRNFLLALIERLDLRN- 11 & 17 \\
\hline & 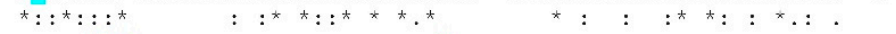 & \\
\hline $\mathrm{Dbe} \triangle \triangle \mathrm{Cl}$ & AYLVAHDWGTALATHLAARRPQLVRGLAFMEF IRPMR-----DWSDFHOHDAARETFRKF 15 & \\
\hline DbeAwt & AYLVAQDWGTALAFHLAARRPQLVRGLAFMEFIRPMR-----DWSDFHQHDAARETFRKF 14 & 7 \\
\hline DhaA & VVLVI HDWGSALGEHWAKRNPERVKGIACMEFIRPIP-----TWDEWPE--EARETFQAF 15 & 52 \\
\hline Lin & VVLVVHDWGSALGIDWARRHRERVQGIAYMEAIAMPI-----EWADFPE--QDRDLFQAF 15 & 54 \\
\hline Dmba & VVLVLHDWGSALGIDWANQHRDRVQGIAFMEAIVTPM-----TWADWPP--AVRGVFQGF 15 & 53 \\
\hline \multirow[t]{2}{*}{ DhlA } & ITLVVQDWGGPLGLTLPMÄDPSRFKRLI IMNACLMTDPVTQPAFSAFVT--QPADGFTAW 17 & 75 \\
\hline & 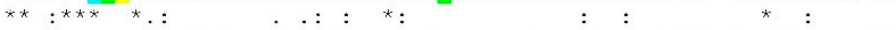 & \\
\hline $\mathrm{Db} \in \mathrm{A} \triangle \mathrm{Cl}$ & RTPGVGEAMILDNNAFVERVLPGSILRTLSEEEMAAYRAPFAT-RESRMPTLMLPRELPI 21 & 1 \\
\hline DbeAwt & RTPGVGEAMILDNNAFVERVLPGSILRTLSEEEMAAYRAPFAT-RESRMPTLMLPRELPI 20 & 0 \\
\hline Dhe & RTADVGREIIIDQNAF IEGVLPKCVVRPLTEVENDHYREPFLK-PVDREPLWRFPNEIPI 21 & 11 \\
\hline LinB & RSQ-AGEELVLQDNVFVEQVLPGLILRPLSEAEMAAYREPFLAAGEARR & 13 \\
\hline DmbA & RSP-QGEPMALEHNIFVERVLPGAILRQLSDEEMNHYRRPFVNGGEDRRPTLSWPRNLPI 21 & 12 \\
\hline \multirow[t]{2}{*}{ DhlA } & KYD-LVTPSDLRLDQFMKRW-----APTLTEAEASAYAAPFPDTSY-QAGVRKFPKMVAQ 22 & 28 \\
\hline & 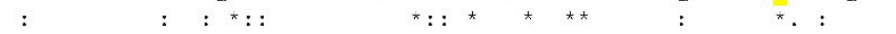 & \\
\hline $\mathrm{DbeA} \triangle \mathrm{Cl}$ & AGEPADVTQALTAAHAALAASTYPKLLFVGSPGALVSPAFAAEFAKTLKHCA-VIQLGAG 26 & 6 \\
\hline & AGEPADVTOALTAAHAALAASTYPKLLFVGSPGALVSPAFAAEFAKTLKHCA-VIQLGAG 26 & 6 \\
\hline & K-TVDIGPG 27 & 7 \\
\hline Lin & AGTPADVVAIARDYAGWLSESPIPKLFINAEPGAITTGR-MRDFCRTWPNQT-EIT-VAG 27 & 70 \\
\hline DmbA & DGEPAEVVALVNEYRSWLEETDMPKLFINAEPGAIITGR-IRDYVRSWPNQT-EIT-VPG 26 & 69 \\
\hline \multirow[t]{2}{*}{ DhlA } & RD-QACIDISTEAISFWQNDWNGQTEMAIGMKDKLLGPDVMYPMKALINGCPEPLEIADA 28 & 87 \\
\hline & . * : $\quad .:: \quad . \quad . \quad:$ & \\
\hline & GHYLQEDHPEAIGRSVAGWIAGIEAASAQREAALEHHHHHH & \\
\hline & SIEAASAQRHAALE------ & \\
\hline DhaA & LHYLQEDNPDLIGSEIARWLPGLA- & \\
\hline Lir & 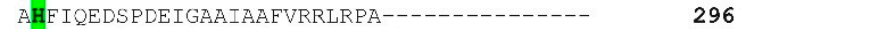 & \\
\hline DmbA & VHFVQEDSPEEIGAAIAQFVRRLRSAAG------------- & \\
\hline \multirow[t]{2}{*}{ DhlA } & GHFVQEFGEQVAREALKHFAETE----------------- & \\
\hline & 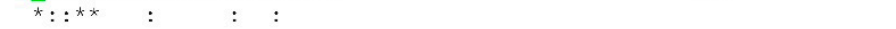 & \\
\hline
\end{tabular}

Figure 4. Multiple sequence alignment of DbeA $\Delta \mathrm{Cl}$ with five Haloalkane dehalogenases (HLDs) deposited in PDB. Amino acids from canonical HLD active site are highlighted in yellow, catalytic triad residues are highlighted in green and second halide binding site residues are highlighted in cyan. Sequence alignment was performed by ClustalW [32].

The molecular structures of DbeA, DhaA, LinB, DmbA, and DhlA were superposed with the DbeA $\Delta \mathrm{Cl}$ enzyme's $\mathrm{C} \alpha$ atoms with root mean square deviations of $0.335,1.018,0.985,1.127$ and $2.112 \AA$, respectively. 3D-superpositions of the DbeA $\Delta \mathrm{Cl}$ structure with the closest similarity model (DhaA) and the lowest identity model (DhlA) are shown in Figure 5a. 


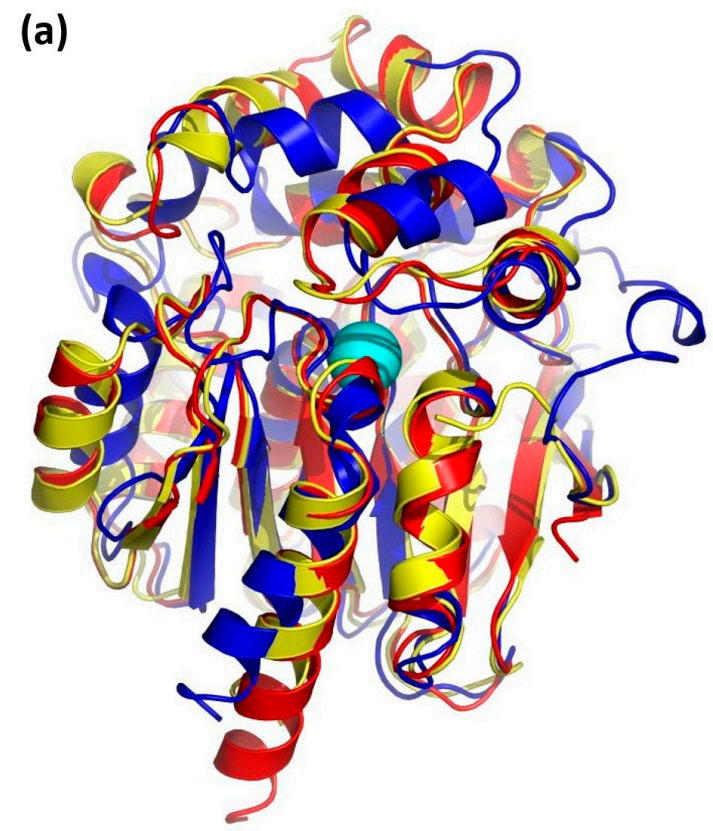

(b) Pro

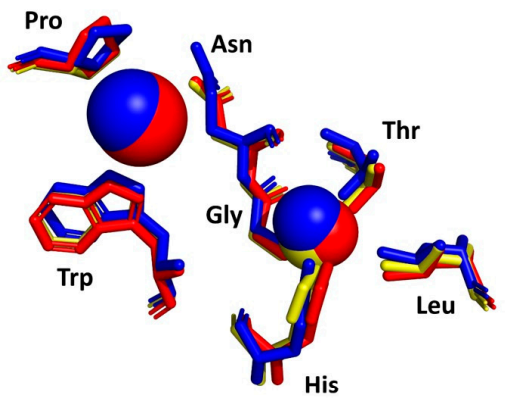

(c)

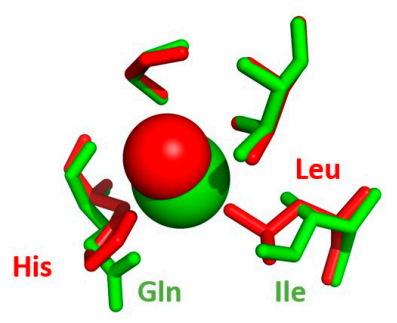

Figure 5. Structural comparison of DbeA $\Delta \mathrm{Cl}$ with homologous dehalogenases. (a) DbeA $\Delta \mathrm{Cl}$ secondary structure superposition with DhaA and DhlA. C $\alpha$ ribbon trace shows elements of the protein secondary structures. The DbeA $\Delta \mathrm{Cl}$ is coloured in red; DhaA is coloured in blue; DhlA is shown in yellow; iodide ion (cyan sphere) is placed in the canonical active site of DbeA $\Delta \mathrm{Cl}$. (b) Superposition of DbeA $\Delta \mathrm{Cl}$, DhaA and DhlA active sites. Amino acids of DbeA $\Delta \mathrm{Cl}$ are shown as red sticks; amino acids of DhaA are shown as blue sticks; amino acids of DhlA are shown as yellow sticks. The iodide ion and water molecule W11 bound to DbeA $\Delta \mathrm{Cl}$ are represented as red spheres; bromide ion and water are shown as blue spheres for the DhaA structure and the single water molecule in the DhlA is shown as yellow sphere. (c) Superposition of second halide binding site residues of mutant and wild type DbeA. DbeA $\Delta \mathrm{Cl}$ amino acids are shown as red sticks; amino acids of DbeAwt are shown as green sticks. Water W71 is shown as red sphere and $\mathrm{Cl}^{-}$ion is shown as green sphere.

In general, the secondary structure elements of the core domain are better conserved compared to the cap domain for the most dehalogenases. Significant differences in cap domains define substrate specificity and variability [1]. The considerable divergence among the superimposed structures was also observable at the relatively disordered $\mathrm{N}$ - and C-terminal parts of the proteins (Figure 5a). The position of the active site residues was well conserved among HLDs with some differences in DhlA structure. DbeA $\Delta \mathrm{Cl}$, DbeA, LinB, DhaA and DmbA belong to the HLD-II subfamily with Asp-His-Glu catalytic triad and Asn-Trp halide binding residues [4] whereas DhlA belongs to the HLD-I subfamily with an Asp-His-Asp + Trp-Trp catalytic pentad composition. The positions of the halide-binding residues Asn 38 and Trp104 in the DbeA $\Delta \mathrm{Cl}$ structure is conserved in comparison to the rest of HLD structures. The position of the catalytic triad (Asp103; His271 and Glu127) is well conserved among the dehalogenases from HLD-II subfamily. The side chain of the catalytic acid Asp in DhlA is situated slightly deeper in the active site cavity in comparison to HLD-II structures.

Superposition of the iodide ion at the DbeA $\Delta \mathrm{Cl}$ active site with halide ions in the vicinity of halide-stabilizing residues of the rest structures reveals some structural differences. DhlA and DmbA contain halide ions inside the active site: $\mathrm{Br}^{-}(\mathrm{DhlA})$ and $\mathrm{Cl}^{-}(\mathrm{DmbA})$ with shifts in 0.47 and $0.39 \AA$ from the DbeA $\triangle \mathrm{Cl}$ iodide ion position, respectively. However, there is only a water molecule in the LinB structure $(0.27 \AA$ away from iodide ion of DbeA $\Delta \mathrm{Cl})$ and an empty space in the DhaA structure (the closest water molecule is located $6.48 \AA$ away from the iodide ion position).

Water molecule W71, which substitutes the halide ion at the second halide-binding site of the DbeA wild type structure, was found at the canonical place where only a water molecule is present in all the HLDs with a shift of $1.39 \AA$ compared to the DhaA structure, $0.80 \AA$ to the DhlA, $0.55 \AA$ to the 
LinB and $0.43 \AA$ to the DmbA, and $1.28 \AA$ away compared to the second chloride anion in the DbeA structure. Superposition of halide binding sites of the DbeA $\Delta \mathrm{Cl}$ structure with halide binding sites of the closest relative (DhaA) and the lowest identity HLD (DhlA) is shown in the Figure 5b.

\section{Conclusions}

After many crystallization experiments and optimization cycles, we found that two point mutations deeply buried in the structure have a significant influence on the crystallization of the macromolecule. Finally, random microseeding experiments with a seed stock prepared from small 3D crystals obtained at a protein concentration of $20 \mathrm{mg} \cdot \mathrm{ml}^{-1}$ and $0.2 \mathrm{M}$ sodium iodide plus $20 \%$ (w/v) PEG 3350 as the precipitant solutions produced crystals of the double mutant DbeA $\Delta \mathrm{Cl}$ from B. elkanii USDA94. These crystals were of sufficient quality for X-ray diffraction experiments. The structure of DbeA $\Delta \mathrm{Cl}$ was solved using molecular replacement and refined to $1.4 \AA$ resolution. Overall, the structure is very similar to other HLDs structures of the $\alpha / \beta$ hydrolase fold superfamily (EC 3.8.1.5). The substitutions Ile44Leu and Gln102His resulted in a space reduction of the second halide-binding site and thus incapability of DbeA $\Delta \mathrm{Cl}$ to bind a second halide anion as compared to the wild type structure. Instead of a chloride anion, a water molecule (W71) was found in the site of which the consequences are, DbeA $\Delta C l$ is less active and less stable in the presence of chloride salts when compared with the DbeAwt enzyme [7]. The DbeA $\Delta \mathrm{Cl}$ structure of B. elkanii was aligned and compared with five molecular structures of haloalkane dehalogenases selected from the PDB. The elements of the secondary structure and the catalytic pentad are well conserved. Superposition of the iodide ion at the DbeA $\Delta \mathrm{Cl}$ active site with the other structures reveals some structural differences. The water molecule W71 located at the compromised second halide-binding site of $\mathrm{DbeA} \Delta \mathrm{Cl}$ was coordinated at the canonical place as compared to other HLDs.

Author Contributions: T.P., J.R.M. and I.K.S. designed the experiments and solved the structure. T.P., B.K., P.G., J.R.M. and M.K. analyzed the data. T.P. and B.K. wrote the manuscript. R.C., J.D., A.M., P.H. and A.S. provided technical support.

Funding: This work was supported by the Grant Agency of the Czech Republic 17-24321S; DAAD mobility grant DAAD-16-09; ERDF project CZ.02.1.01/0.0/0.0/15_003/0000441; Ministry of Education, Youth and Sports of the Czech Republic (CZ.1.05/2.1.00/01.0024, CZ.1.05/2.1.00/01.0001, and LM2015055); GAJU 17/2019/P.

Acknowledgments: The diffraction data were collected on the beam line MX14.1 at the BESSY II electron storage ring operated by the Helmholtz-Zentrum Berlin. We would particularly like to acknowledge the help and support of Manfred S. Weiss during data collection. Also, we would like to thank Stefan A. Kolek (Douglas Instruments Ltd, Hungerford, UK) for providing crucial information about the random seeding experiment during the 2014 FEBS-Instruct practical course PC14-005 at Nove Hrady, Czech Republic.

Conflicts of Interest: No interest conflict exists among the authors.

\section{References}

1. Newman, J.; Peat, T.S.; Richard, R.; Kan, L.; Swanson, P.E.; Affholter, J.A.; Holmes, I.H.; Schindler, J.F.; Unkefer, C.J.; Terwilliger, T.C. Haloalkane dehalogenases: Structure of a Rhodococcus enzyme. Biochemistry 1999, 38, 16105-16114. [CrossRef] [PubMed]

2. Janssen, D.B.; Dinkla, I.J.T.; Poelarends, G.J.; Terpstra, P. Bacterial degradation of xenobiotic compounds: Evolution and distribution of novel enzyme activities. Environ. Microbiol. 2005, 7, 1868-1882. [CrossRef] [PubMed]

3. Prokop, Z.; Sato, Y.; Brezovsky, J.; Mozga, T.; Chaloupkova, R.; Koudelakova, T.; Jerabek, P.; Stepankova, V.; Natsume, R.; van Leeuwen, J.G.; et al. Enantioselectivity of haloalkane dehalogenases and its modulation by surface loop engineering. Angew. Chem. Int. Ed. Engl. 2010, 49, 6111-6115. [CrossRef] [PubMed]

4. Chovancova, E.; Kosinski, J.; Bujnicki, J.M.; Damborsky, J. Phylogenetic analysis of haloalkane dehalogenases. Proteins 2007, 62, 305-306. [CrossRef] [PubMed]

5. Holmquist, M. Alpha/beta-hydrolase fold enzymes: Structures, functions and mechanisms. Curr. Protein Pept. Sci. 2000, 1, 209-235. [CrossRef] [PubMed] 
6. Prokop, Z.; Oplustil, F.; DeFrank, J.; Damborsky, J. Enzymes fight chemical weapons. Biotechnol. J. 2006, 1, 1370-1380. [CrossRef]

7. Chaloupkova, R.; Prudnikova, T.; Rezacova, P.; Prokop, Z.; Koudelakova, T.; Daniel, L.; Brezovsky, J.; Ikeda-Ohtsubo, W.; Sato, Y.; Kuty, M.; et al. Structural and functional analysis of a novel haloalkane dehalogenase with two halide-binding sites. Acta Cryst. 2014, 70, 1884-1897. [CrossRef]

8. Berman, H.M.; Westbrook, J.; Feng, Z.; Gilliland, G.; Bhat, T.N.; Weissig, H.; Shindyalov, I.N.; Bourne, P.E. The Protein Data Bank. Nucleic Acids Res. 2000, 28, 235-242. [CrossRef]

9. Ducruix, A.; Giegé, R. Crystallization of Nucleic Acids and Proteins; Oxford University Press: Oxford, UK, 1999. [CrossRef]

10. Chayen, N.E.J. Crystallization with oils: A new dimension in macromolecular crystal growth. J. Cryst. Growth 1999, 196, 434-441. [CrossRef]

11. Bergfors, T.M. Protein Crystallization: Techniques, Strategies and Tips; International University Line: La Jolla, CA, USA, 1999. [CrossRef]

12. Gavira, J.A.; Jesus, W.; Camara-Artigas, A.; Lopez-Garriga, J.; Garcia-Ruiz, J.M. Crystallization and diffraction patterns of the oxy and cyano forms of the Lucina pectinata haemoglobins complex. Acta Cryst. 2006, 62, 196-199. [CrossRef]

13. Gerlach, M.; Mueller, U.; Weiss, M.S. The MX beamlines BL14.1-3 at BESSY II. JLSRF 2016, 2, 1-6. [CrossRef]

14. Sparta, K.M.; Krug, M.; Heinemann, U.; Mueller, U.; Weiss, M.S. XDSAPP2.0. J. Appl. Cryst. 2016, 49, 1085-1092. [CrossRef]

15. Kabsch, W. Automatic processing of rotation diffraction data from crystals of initially unknown symmetry and cell constants. J. Appl. Cryst. 1993, 26, 795-800. [CrossRef]

16. Vagin, A.; Teplyakov, A. MOLREP: An automated program for Molecular Replacement. J. Appl. Cryst. 1997, 30, 1022-1025. [CrossRef]

17. Murshudov, G.N.; Skubak, P.; Lebedev, A.A.; Pannu, N.S.; Steiner, R.A.; Nicholls, R.A.; Winn, M.D.; Long, F.; Vagin, A.A. REFMAC5 for the refinement of macromolecular crystal structures. Acta Cryst. 2011, 67, 355-367. [CrossRef]

18. Emsley, P.; Lohkamp, B.; Scott, W.G.; Cowtan, K. Features and development of Coot. Acta Cryst. 2010, 66, 486-501. [CrossRef]

19. Winn, M.D.; Ballard, C.C.; Cowtan, K.D.; Dodson, E.J.; Emsley, P.; Evans, P.R.; Keegan, R.M.; Krissinel, E.B.; Leslie, A.G.W.; McCoy, A.; et al. Overview of the CCP4 suite and current developments. Acta Cryst. 2011, 67, 235-242. [CrossRef]

20. Chen, V.B.; Arendall, W.B.; Headd, J.J.; Keedy, D.A.; Immormino, R.M.; Kapral, G.J.; Murray, L.W.; Richardson, J.S.; Richardson, D.C. MolProbity: All-atom structure validation for macromolecular crystallography. Acta Cryst. 2010, 66, 12-21. [CrossRef]

21. Hintze, B.J.; Lewis, S.M.; Richardson, J.S.; Richardson, D.C. Molprobity's ultimate rotamer-library distributions for model validation. Proteins 2016, 84, 1177-1189. [CrossRef]

22. Gore, S.; Velankar, S.; Kleywegt, G.J. Implementing an X-ray validation pipeline for the Protein Data Bank. Acta Cryst. 2012, 68, 478-483. [CrossRef]

23. Schrodinger, L.L.C. The PyMOL Molecular Graphics System, Version 2.0. 2019. Available online: https: //pymol.org/2/ (accessed on 23 July 2019).

24. Prudnikova, T.; Mozga, T.; Rezacova, P.; Chaloupkova, R.; Sato, Y.; Nagata, Y.; Brynda, J.; Kuty, M.; Damborsky, J.; Kuta-Smatanova, I. Crystallization and Preliminary X-ray Analysis of a Novel Haloalkane Dehalogenase DbeA from Bradyrhizobium elkani USDA94. Acta Cryst. 2009, 65, 353-356. [CrossRef]

25. Shaw Stewart, P.D.; Kolek, S.A.; Briggs, R.A.; Chayen, N.E.; Baldock, P.F.M. Random Microseeding: A Theoretical and Practical Exploration of Seed Stability and Seeding Techniques for Successful Protein Crystallization. Cryst. Growth Des. 2011, 11, 3432-3441. [CrossRef]

26. Ollis, D.L.; Cheah, E.; Cygler, M.; Dijkstra, B.; Frolow, F.; Franken, S.M.; Harel, M.; Remington, S.J.; Silman, I.; Schrag, J.; et al. The alpha/beta hydrolase fold. Protein Eng. 1992, 5, 197-211. [CrossRef] [PubMed]

27. Krissinel, E.; Henrick, K. Inference of macromolecular assemblies from crystalline state. J. Mol. Biol. 2007, 372, 774-797. [CrossRef] [PubMed]

28. Pikkemaat, M.G.; Ridder, I.S.; Rozeboom, H.J.; Kalk, K.H.; Dijkstra, B.W.; Janssen, D.B. Crystallographic and kinetic evidence of a collision complex formed during halide import in haloalkane dehalogenase. Biochemistry 1999, 38, 12052-12061. [CrossRef] [PubMed] 
29. Marek, J.; Vevodova, J.; Smatanova, I.K.; Nagata, Y.; Svensson, L.A.; Newman, J.; Takagi, M.; Damborsky, J. Crystal structure of the haloalkane dehalogenase from Sphingomonas paucimobilis UT26. Biochemistry 2000, 39, 14082-14086. [CrossRef]

30. Mazumdar, P.A.; Hulecki, J.C.; Cherney, M.M.; Garen, C.R.; James, M.N.G. X-ray crystal structure of Mycobacterium tuberculosis haloalkane dehalogenase Rv2579. Biochim. Biophys. Acta. 2008, 1784, 351-362. [CrossRef]

31. Koudelakova, T.; Bidmanova, S.; Dvorak, P.; Pavelka, A.; Chaloupkova, R.; Prokop, Z.; Damborsky, J. Haloalkane dehalogenases: Biotechnological applications. Biotechnol. J. 2013, 8, 32-45. [CrossRef]

32. Larkin, M.A.; Blackshields, G.; Brown, N.P.; Chenna, R.; McGettigan, P.A.; McWilliam, H.; Valentin, F.; Wallace, I.M.; Wilm, A.; Lopez, R.; et al. Clustal W and Clustal X version 2.0. Bioinformatics 2007, 23, 2947-2948. [CrossRef]

(C) 2019 by the authors. Licensee MDPI, Basel, Switzerland. This article is an open access article distributed under the terms and conditions of the Creative Commons Attribution (CC BY) license (http://creativecommons.org/licenses/by/4.0/). 